\title{
Gas temperature and excitation classes in planetary nebulae
}

\author{
L. P. Martins and S. M. Viegas
}

Instituto de Astronomia, Geofísica e Ciências Atmosféricas, São Paulo, Brazil

Received 2 November 2001 / Accepted 25 March 2002

\begin{abstract}
Empirical methods to estimate the elemental abundances in planetary nebulae usually use the temperatures derived from the $[\mathrm{O} \mathrm{III}]$ and $[\mathrm{N} \mathrm{II}]$ emission-line ratios, respectively, for the high- and low-ionization zones. However, for a large number of objects these values may not be available. In order to overcome this difficulty and allow a better determination of abundances, we discuss the relationship between these two temperatures. Although a correlation is not easily seen when a sample of different PNe types is used, the situation is improved when they are gathered into excitation classes. From [OII]/[OIII] and HeII/HeI line ratios, we define four excitation classes. Then, using standard photoionization models which fit most of the data, a linear relation between the two temperatures is obtained for each of the four excitation classes. The method is applied to several objects for which only one temperature can be obtained from the observed emission lines and is tested by recalculation of the radial abundance gradient of the Galaxy using a larger number of PNe. We verified that our previous gradient results, obtained with a smaller sample of planetary nebulae, are not changed, indicating that the temperature relation obtained from the photoionization models are a good approximation, and the corresponding statistical error decreases as expected.
\end{abstract}

Key words. stars: abundances - ISM: abundances - planetary nebulae: general

\section{Introduction}

The most common empirical method used to determine chemical abundances of photoionized nebulae is based on the observed intensities of forbidden emission lines (Peimbert \& Costero 1969). This method depends on the determination of the electronic density and temperature. The nebula is assumed to have 2 regions: an outer lowionization zone and an inner high-ionization zone, respectively characterized by the ions $\mathrm{N}^{+}$and $\mathrm{O}^{++}$. Each zone has a characteristic temperature, respectively, $T_{\mathrm{NII}}$ and $T_{\text {OIII. }}$ Following the ionization potentials, the ions are assumed to be present in one or another zone, as indicated by photoionization models. The ionic abundances of $\mathrm{N}^{+}$, $\mathrm{O}^{+}, \mathrm{S}^{+}$and $\mathrm{S}^{++}$are calculated using $T_{\mathrm{NII}}$, while $T_{\mathrm{OII}}$ is used for $\mathrm{O}^{++}$and $\mathrm{Ne}^{++}$. The electronic density usually comes from the [S II] line ratio.

One of the main problems in determining chemical abundances of planetary nebulae (PNe) using the forbidden lines is the calculation of both electronic temperatures $T_{\mathrm{NII}}$ and $T_{\mathrm{OIII}}$. These temperatures are derived from the ratio between the auroral and nebular emissionlines of $\mathrm{O}^{++}$and $\mathrm{N}^{+}$. The faint auroral lines [OIII] $\lambda 4363$ and [NII] $\lambda 5755$ are in the bluer part of the spectra, where the detectors are generally less sensitive. When one of

\footnotetext{
Send offprint requests to: L. P. Martins,

e-mail: lucimara@iagusp.usp.br

* Tables 3-5, 7 and 9 are only available in electronic form at http://www.edpsciences.org
}

these lines is not observed, it is very common, in the literature, to assume that either the temperatures of the highand low-ionization zones are equal, or the gas temperature is $10^{4} \mathrm{~K}$. This may be a rough approximation, and may induce serious errors in the abundance determinations, since they are very sensitive to the electronic temperature. In addition, such an approximation can lead to false conclusions drawn from a sample of PNe abundances as, for instance, those regarding the radial abundance gradient of the Galaxy or other aspects of galactic chemical evolution.

For many objects, $T_{\mathrm{OIII}}$ can be obtained, but not $T_{\mathrm{NII}}$. In the case of empirical abundance determinations for HII regions, the missing temperature problem was overcome by Pagel (1992). These authors found a relation between $T_{\text {OIII }}$ and $T_{\text {OII }}$ (temperatures of the high and low ionization zones), based on photoionization models of Stasinska (1990).

Here we discuss an alternative method to determine the missing temperature for planetary nebulae. We verified that such a method could be obtained as long as the $\mathrm{PNe}$ are separated into excitation classes (Sect. 2). We then look for a relation between the temperatures of the high- and low-ionization zones using photoionization models. The calculated $T_{\mathrm{OIII}}$ and $T_{\mathrm{NII}}$ values are compared to the temperatures derived from observations of a sample of planetary nebulae of a given excitation class (Sect. 3). The temperature relation, deduced from a grid of photoionization models, is applied to other PNe for which one of the temperatures is missing, and the results are tested by 


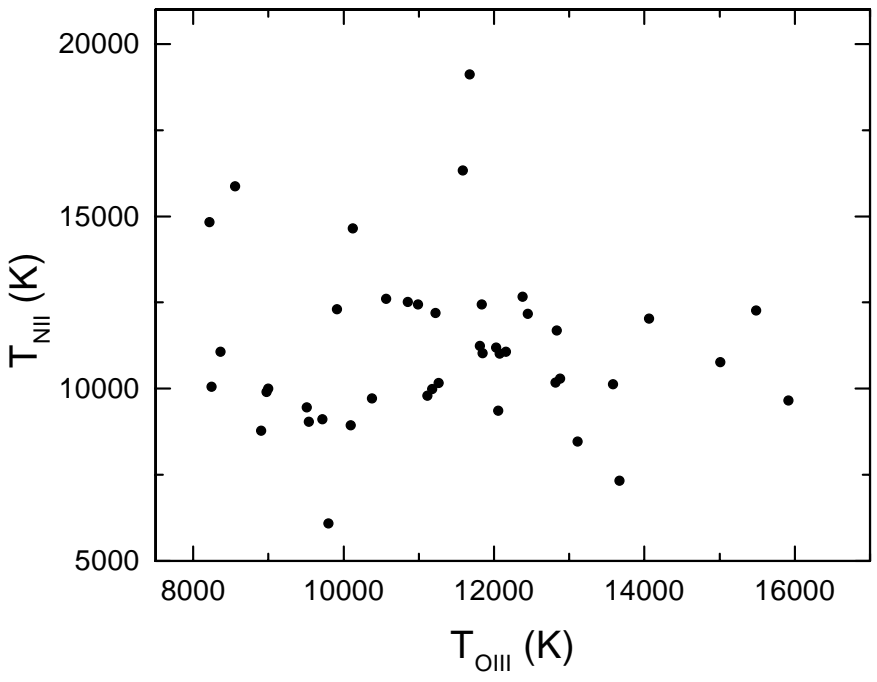

Fig. 1. $T_{\mathrm{OIII}}$ versus $T_{\mathrm{NII}}$ for type II PNe. Figure shows that no correlation exists between these two temperatures. The correlation coefficient is 0.21 .

recalculating the radial abundance gradient of the Galaxy (Sect. 4). The concluding remarks appear in Sect. 5 .

\section{The excitation classes}

In order to find a relation between the high- and lowionization zones of planetary nebulae, data from a sample of type II PNe (Martins \& Viegas 2000) were plotted. As seen in Fig. 1, no correlation is found between $T_{\text {OIII }}$ and $T_{\mathrm{NII}}$. However, any possible correlation could have been destroyed because the sample includes objects of different excitation classes. In order to verify the importance of the excitation class in defining the temperature correlation, in the following a classification of PNe into excitation classes is presented.

Since 1864, when Huggins showed bright line spectra of nebulae, several classification schemes based on their spectra have been suggested. Cannon (1916) proposed the first one, based on the HeII $\lambda 4686$ emission-line. Later, Page (1942) divided the PNe spectra in classes from 1 to 8, using the $[\mathrm{OII}] \lambda 3727 /[\mathrm{OIII}] \lambda 4959$ and HeII $\lambda 4686 / \mathrm{H} \beta$ line ratios respectively for low- and high-ionization nebulae. A more refined classification was proposed by Aller (1956). His criteria are similar to Page's, based on the $[\mathrm{OIII}] \lambda 5007 / \mathrm{H} \beta$ and $\mathrm{He}$ II $\lambda 4686 / \mathrm{H} \beta$ line ratios and largely used to classify the planetary nebulae.

The excitation classes are defined using the stronger lines of the nebular spectra. These lines depend on the central star temperature, as well as the central star luminosity, nebular density, geometry and abundances. To avoid the dependence of the excitation classes on this last parameter, the classification criteria based on line ratios involving different elements should not be used (Ratag et al. 1997).

It is impossible to associate excitation classes with a single parameter of the nebulae. Gurzadyan \& Egikyan (1991) related the excitation classes to nebular sizes, and
Table 1. Planetary nebulae excitation classes.

\begin{tabular}{ccc}
\hline \hline Class 1 & $R 1 \leq 1$ & - \\
Class 2 & $1 \leq R 1 \leq 8$ & $R 2 \geq 1$ \\
Class 3 & $1 \leq R 1 \leq 8$ & $R 2 \leq 1$ \\
Class 4 & $R 1 \geq 8$ & - \\
\hline
\end{tabular}

Table 2. Relation between excitation classes.

\begin{tabular}{ccc}
\hline \hline Adopted Classification & Webster 1988 & Aller 1956 \\
\hline Class 1 & Classes 2-4 & Classes 3-5 \\
Class 2 & Class 4 & Classes 4-5 \\
Class 3 & Classes 4-8 & Classes 5-7 \\
Class 4 & Class 9 & Classes 8-10 \\
\hline
\end{tabular}

consequently, to the evolutionary stage of the planetary nebula. On the other hand, comparing excitation class distributions in different locations "such as each of the Magellanic Clouds, the galactic bulge and kinematic subgroups in the solar neighborhood", Webster (1988) concluded that they are a function of some fundamental property of the object rather than just a consequence of the stage of evolution. Therefore, when studying PNe, excitation classes may be a very convenient independent parameter (Gurzadyan \& Egikyan 1991).

In this work we follow the suggestion of Ratag et al. (1997), i.e., we use the following line ratios:

$R 1=\frac{\operatorname{HeII} \lambda 4686}{\operatorname{HeI} \lambda 5876}$ and $R 2=\frac{[\mathrm{OII}] \lambda 3727}{[\mathrm{OIII}] \lambda \lambda 4959,5007}$.

Essentially we can separate low, intermediate and high excitation classes using the helium line ratio $(R 1)$, whereas the oxygen line ratio $(R 2)$ can be used to refine the classification. As shown in Table 1 , following the $R 1$ and $R 2$ values four classes are defined in this work.

Class 1 includes low excitation objects, classes 2 and 3 contain intermediate excitation objects whereas class 4 , high excitation objects. These classes are compared to those proposed by other authors in Table 2 .

The criteria listed above are used to classify types I-III planetary nebulae from a sample of observed objects (Tables 3-5). The PN name is listed in Col. 1, the references for the line intensities appear in Col. 2, the electronic density, excitation class, and electronic temperatures are given respectively in Cols. 3 to 6 , while the chemical abundances of oxygen, nitrogen, sulfur and neon, in Cols. 7 to 10 . The abundances were calculated by the empirical method, as described by Martins \& Viegas (2000). For some objects the [OII] $\lambda 3727$ line was not observed, not only because it is a weak line, but also because the blue part of the line spectra is not available. In these cases, the low excitation objects are separated from the others using only $R 2$. For the intermediate excitation nebulae, the Aller (1956) criteria is applied, according to the comparison shown in Table 2. There are objects for which neither [OII] $\lambda 3727$ nor HeII $\lambda 4686$ are available. In theses cases, 
Aller criteria are used. These objects are identified by an asterisk in the Tables $3-5$.

For each type of planetary nebula, the number of objects in each excitation class is not large enough to provide a good relation between $T_{\text {OIII }}$ and $T_{\text {NII }}$ (Figs. 2-4). A theoretical relation can be established using photoionization models, as discussed below.

\section{Photoionization models}

The photoionization code AANGABA (Gruenwald \& Viegas 1992) is used to model a spherically symmetric nebula, photoionized by a black body ionizing radiation characterized by the temperature and luminosity of the central star.

To account for the large range of observed physical parameters of the planetary nebulae, a grid of models is obtained for the stellar temperature, stellar luminosity and gas density varying, respectively, in the ranges: 40000 to $190000 \mathrm{~K}, 500$ to $50000 \mathrm{~L} / L_{\odot}$ and 200 to $10000 \mathrm{~cm}^{-3}$. The elemental abundances adopted are: $\mathrm{He}=0.115$, $\mathrm{N}=2.24 \times 10^{-4}, \mathrm{Ne}=1.23 \times 10^{-4}, \mathrm{Mg}=3.8 \times 10^{-7}$, $\mathrm{Si}=3.5 \times 10^{-7}, \mathrm{~S}=8.32 \times 10^{-6}, \mathrm{Cl}=3.2 \times 10^{-9}, \mathrm{Ar}=$ $2.45 \times 10^{-6}$ and $\mathrm{Fe}=4.7 \times 10^{-7}$ (Kingsburgh \& Barlow 1994). However, different carbon and oxygen abundances are adopted, in order to reach higher temperatures. Our grid includes models with $1.6 \times 10^{-4} \leq \mathrm{C} / \mathrm{H} \leq 5.5 \times 10^{-4}$ and $1.5 \times 10^{-4} \leq \mathrm{O} / \mathrm{H} \leq 4.79 \times 10^{-4}$. The upper values come from Kingsburgh \& Barlow (1994), whereas the lower ones are chosen from the $\mathrm{PNe}$ abundances in our sample.

The physical conditions are calculated in several shells. The calculations start at the nebula inner radius and stop at an outer shell where hydrogen is neutral $(\mathrm{HII} / \mathrm{H} \leq$ $\left.10^{-4}\right)$ i.e., when the optical depth at the Lyman limit, $\tau_{\mathrm{Ly}}$, is much larger than unity. However, some planetary nebulae could be matter-bound (see, for instance, Gruenwald \& Viegas 2000). In this case, at the $\mathrm{PN}$ outer radius $\mathrm{H}$ is not completely neutral, $\tau_{\mathrm{Ly}}$ is smaller, and the average physical conditions of the emitting gas differ from the radiation-bound case. In order to simulate matter-bound planetary nebulae, models with $0.5 \leq \tau_{\text {Ly }} \leq 100$ have also been built.

\subsection{Results}

Around 700 models were generated, combining the input parameters. Following the theoretical $R 1$ and $R 2$ line ratios, the models are classified in the excitation classes according to the criteria listed in Table 1. The corresponding values for $T_{\mathrm{OIII}}$ and $T_{\mathrm{NII}}$ are compared to the observational data for planetary nebulae of types I-III in Figs. 2-4, respectively. The PNe that, even accounting for the uncertainties, show a large disagreement with the models are labeled in these figures.

A certain scatter of the observational points relative to the theoretical results is expected because the models are
Table 6. Linear fit to the temperature relation ${ }^{[*]}$.

\begin{tabular}{|c|c|c|c|c|}
\hline & $a$ & $\sigma_{a}$ & $\bar{b}$ & $\overline{\sigma_{b}}$ \\
\hline Class 1 & 0.77 & 0.03 & 2782 & 316 \\
\hline Class 2 & 0.80 & 0.11 & 1653 & 121 \\
\hline Class 3 & 0.50 & 0.02 & 5129 & 244 \\
\hline Class 4 & 0.92 & 0.01 & 545 & 12 \\
\hline
\end{tabular}

extremely idealized: the PNe are supposed to be spherically symmetric with constant density. Therefore, accounting for the observational errors, we can say that model results roughly reproduce the behavior of $T_{\text {OIII versus }} T_{\text {NII }}$. Notice, however, that for several high $T_{\text {OIII }}$ class 3 and 4 $\mathrm{PNe}$ a theoretical relation based on the photoionization models may not provide a good result for the missing temperature.

For each excitation class, and independent of the type of the PNe, a linear least square fit of the model results is obtained, where $T_{\mathrm{NII}}=a T_{\mathrm{OIII}}+b$. The $a$ and $b$ coefficients, as well as the corresponding uncertainties, are listed in Table 6 .

As seen in Fig. 2, most of the class 3 type I PNe with $T_{\text {OIII }}<1.4 \times 10^{4} \mathrm{~K}$ are clustered below the $T_{\text {NII }}$ coming from the models, and for 5 out of 9 the temperatures do not reach the theoretical values even accounting for the data errors. On the other hand, the data of the PNe with $T_{\text {OIII }}>1.4 \times 10^{4} \mathrm{~K}$ are not reproduced by the models. A similar problem occurs for the class 4 types I-III PNe (Figs. 2-4).

Regarding class 3 of type II ( 3 objects) and class 2 of type III PNe (2 objects), within the errors the data are explained by the models. Notice that there are no observational data available for excitation class 3 of type III planetary nebulae.

Another interesting point is that in general $T_{\mathrm{NII}}$ is lower than $T_{\text {OIII }}$ for the $\mathrm{PNe}$ in the sample, following the trend of the model results. However, only 6 out of 17 class 1 type II PNe show $T_{\mathrm{NII}}<T_{\mathrm{OIII}}$. Although the model results are within the errors, the discrepancy between observational data and theoretical results may indicate that standard photoionization models are not a good approach to class 1 type II PNe. It is well known that a hard ionizing radiation, which is mainly absorbed at the low-ionization zone, can lead to $T_{\mathrm{NII}}>T_{\mathrm{OIII}}$. However, class $1 \mathrm{PNe}$ are low excitation objects, and we expect that the ionizing star temperature is not very high, so that not enough high energy photons reach the nebula. In this case, the discrepancy between observations and model results may be due to shocks or to density condensations, which are not accounted for in the standard models used in this paper. 

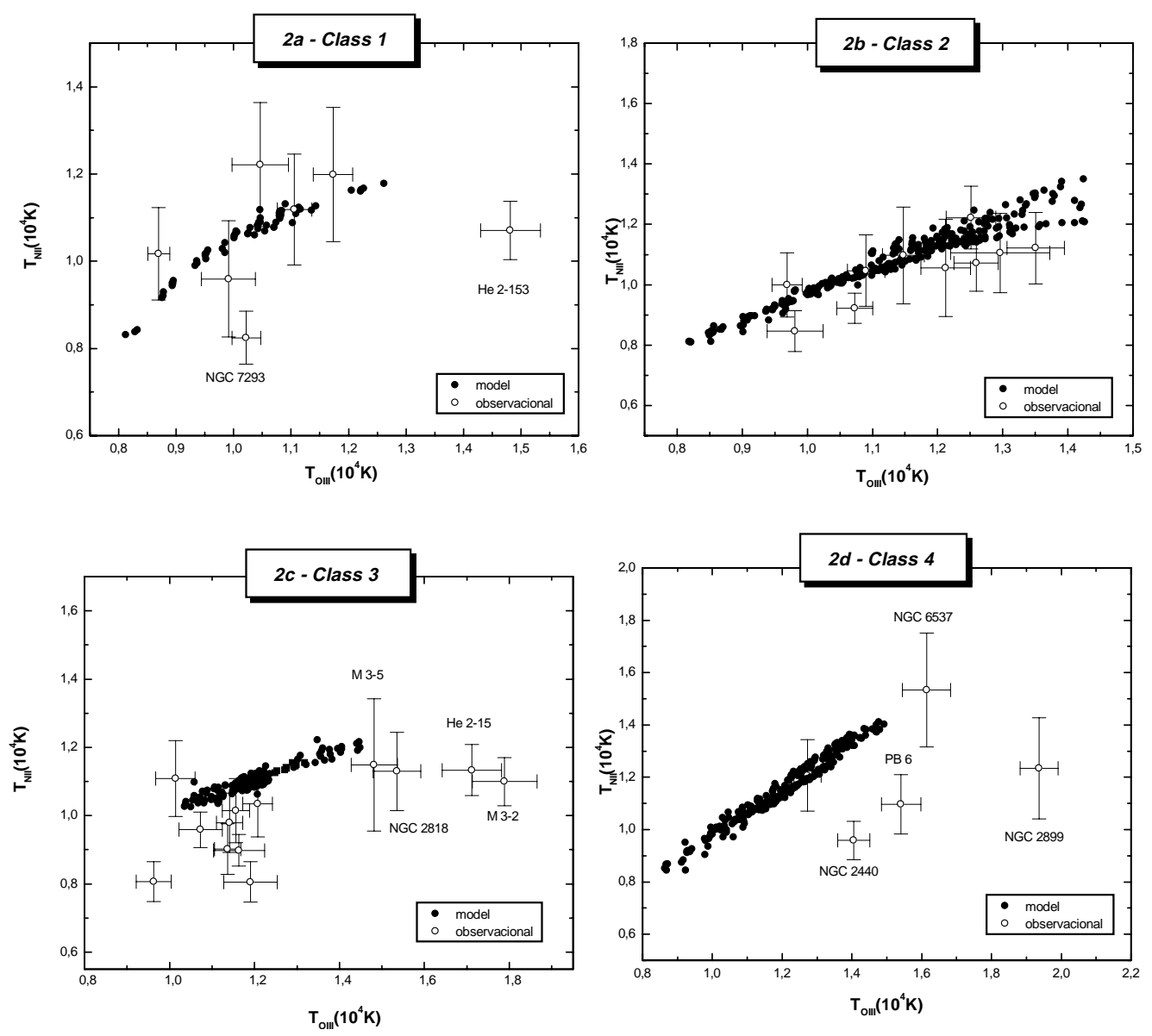

Fig. 2. Theoretical and observational $T_{\text {OIII }}$ versus $T_{\text {NII }}$. The filled circles are the model results and the open circles are the type I PNe with the required emission lines observed.

\subsection{Objects with special features}

For several PNe in our sample there is a disagreement between observed and theoretical results. For some of them, a search in the literature has shown that these objects have special features, as described below.

Regarding type I PNe (Fig. 2), class 1 NGC 7293 is a singular planetary nebula, with a system of ionized knots showing comet like tails (Meaburn et al. 1996) and He 2-153 may also have been missclassified, since following Acker et al. (1989) it should be class 3. In addition, class 3 NGC 2818 could have filaments and condensations (Phillips \& Cuesta 1998) and He 2-15 is highly enriched in He and N (Kaller et al. 1996). Nothing special was found in the literature about the class 3 M3-5 and M3-2. We suspect that they could also have some especial features that could explain the discrepancies with model results, although some contribution from observational errors cannot be excluded. In fact, it is worth noting that type I NGC 6537 is classified as class 2 PN if the emission-line intensities of Freitas-Pacheco et al. (1992) are used, while it is a class 4 object with Aller et al. (1995) data. Using the two samples of data, its high- and low-ionization zone temperatures change by less than $10 \%$. Nevertheless, as a class $2 \mathrm{PN}$ it would have $T_{\mathrm{NII}}>T_{\mathrm{OIII}}$ and would be outside the region covered by the models in Fig. 2b, while as class 4 its location (Fig. 2d) indicates accordance with the trend showed by the model results. As pointed out by Hyung (1999), NGC 6537 is an extremely high excitation object, in agreement with class 4 , under the influence of strong stellar winds and shock heating, which could explain $T_{\mathrm{NII}}$ and $T_{\mathrm{OIII}}$ slightly higher $(<8 \%)$ than the maximum temperatures found with standard photoionization models.

Type II class 2 planetary nebula NGC 2392 "possesses an extremely unusual spectro-kinematic morphology characterized by several strange and elusive structures" (Phillips \& Cuesta 1999), while following Tamura \& Shaw (1987), type III class 1 PN K 3-67 "is an interesting class of $\mathrm{PN}$, which should be classified as population II kinematically, but which show $\mathrm{He}$ and $\mathrm{N}$ enrichment.

For all types of planetary nebulae, class 4 objects show larger discrepancies between models and the observational data, even accounting for the observational errors. The discrepancies could be due to extreme physical conditions prevailing in these objects, which cannot be reproduced by the simple models presented here.

To illustrate this point, let us comment on some singular class 4 type II planetary nebulae. NGC 2371 presents some peculiarities in its spectra that need a more careful investigation. This object is probably close to the blue 

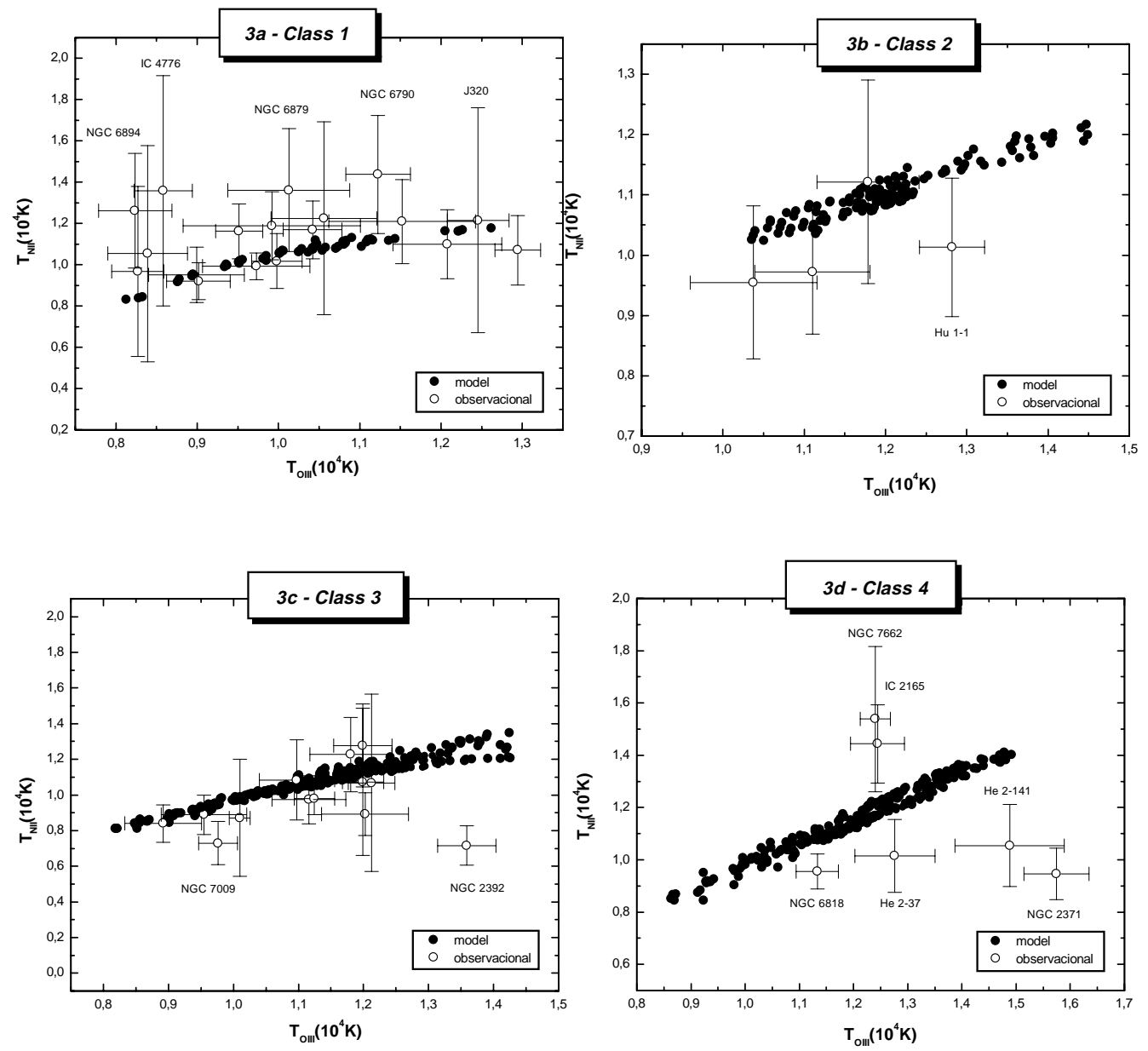

Fig. 3. Theoretical and observational $T_{\mathrm{OIII}}$ versus $T_{\mathrm{NII}}$. The filled circles are the model results and the open circles are type II PNe with the required emission lines observed.

bend of the post-AGB evolutionary sequence and shows prominent $\mathrm{O}$ VI emissions with (so far) unique double narrow and broad features (Kaler et al. 1993). NGC 7662 shows evidence for anomalous low-ionization filaments in a high-ionization substract (Balick 1987). NGC 6818 shows an uncommon kinematic structure (Sabbadin 1984). High dispersion spectra show complicated line profile and splitting correlated, indicating that intrincated internal movements are superposed to the expansion pattern (Hyung et al. 1999a).

From these examples we may infer that several PNe have special characteristics that cannot be explained by general, simplified models. For these objects, specific, more sophisticated models, which are out of the scope of this paper, should probably be used to reproduce the gas physical conditions.

\section{Testing the temperature relation}

The calculation of the radial abundance gradient of the galaxy can provide a simple test for the theoretical relation between $T_{\mathrm{OIII}}$ and $T_{\mathrm{NII}}$ found above. In a previous paper, we discussed the radial abundance gradients using type II planetary nebulae (Martins \& Viegas 2000).
However, objects for which one of those temperatures could not be deduced from the observations were discarded from the sample. Now we can use the high- and lowionization zone temperature relations (Sect. 3) to recalculate the elemental abundances of these objects, include them in the sample and recalculate the abundance gradient. If those temperature relations obtained from photoionization models are a good approach to the PNe empirical temperatures, we expect that adding those objects to our previous PNe sample (Tables 3-5), the new abundance gradient would agree with our previous value of the gradient (Martins \& Viegas 2000). In fact, such an agreement would indicate that the temperature relations based on the excitation classes are not introducing errors on the empirical abundances.

\subsection{Type I/ planetary nebulae}

In order to obtain the radial abundance gradient, type II planetary nebulae have been used because they are more representatives of the galactic disc and a larger sample is available in the literature. The abundances of oxygen, neon sulfur and nitrogen are calculated from the published emission-line intensities (see Martins \& Viegas 2000). 

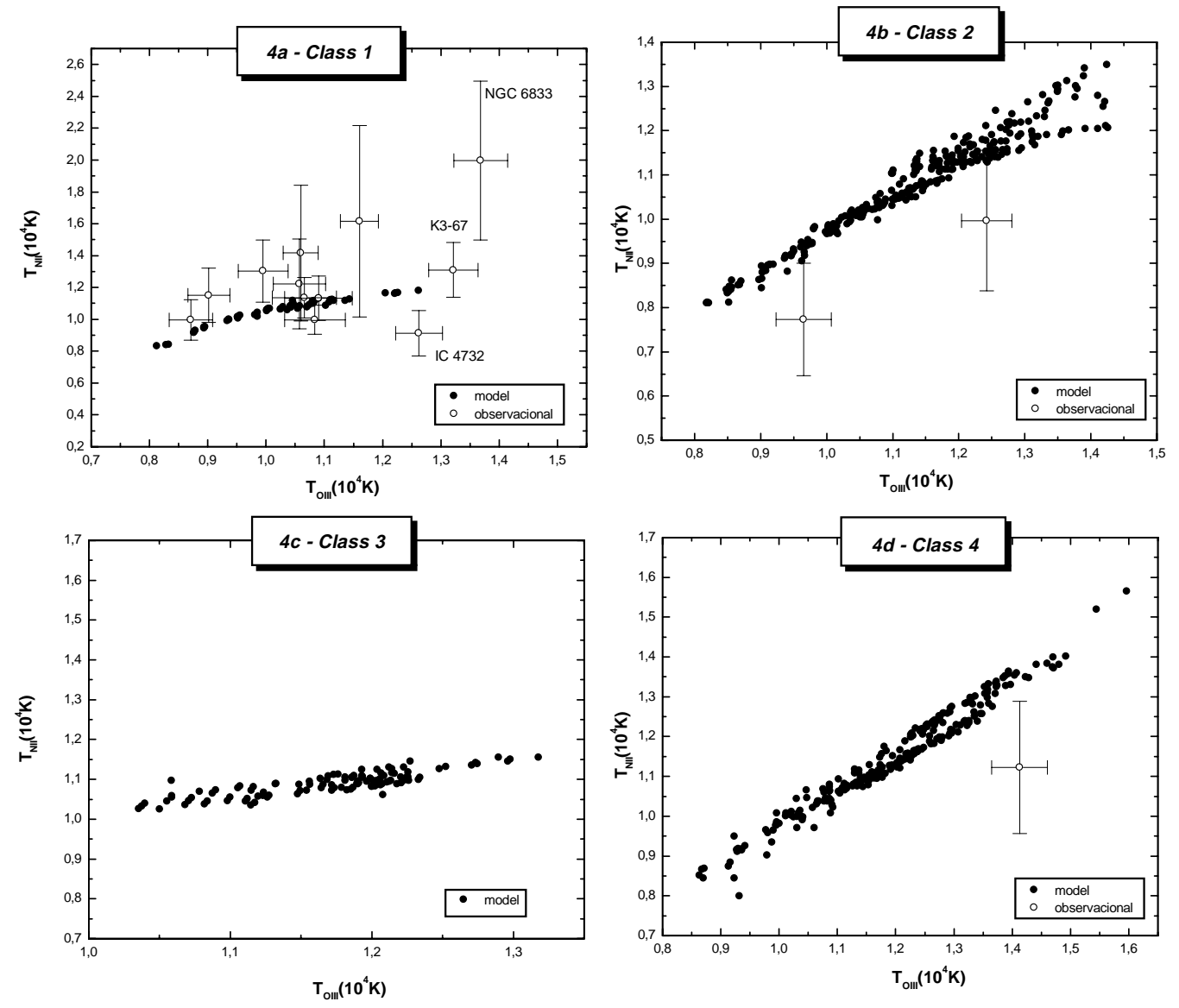

Fig. 4. Theoretical and observational $T_{\mathrm{OIII}}$ versus $T_{\mathrm{NII}}$. The filled circles are the model results and the open circles are the type III PNe with the required emission lines observed.

The distances come from Maciel \& Quireza (1999). These authors used the distances obtained by Maciel (1984), but changed the sun distance to $7.6 \mathrm{kpc}$, a more recent value. Maciel (1984) used a statistical method based on an empirical relation between the mass and the radius of the nebulae. The uncertainty in the distances may be even larger than $50 \%$, leading to a large scatter of the data, and so, to a smaller value of the gradient (Maciel \& Quireza 1999). Thus a better determination of the distances would be important for studies on the chemical evolution of galaxies, but does not affect our conclusions relative to the relation between the temperatures of the high- and low-ionization zones.

Type II planetary nebulae with observed emissionlines allowing to deduce the electron density and either the high- or the low-ionization temperature were gathered from the literature and divided into the excitation classes following the criteria listed in Table 1 . Then, using the least square linear fit coefficients given in Table 6 , the missing temperature is obtained and the elemental abundances are deduced for these objects.

The results are shown in Table 7, where the PN name is listed in Col. 1, the references regarding the emissionline intensities appear in Col. 2, the excitation class and electronic density are given respectively in Cols. 3 and 4, while the temperatures for high- and low-ionization zones are listed in Cols. 5 and 6 . The empirical abundances are shown in Cols. 7 to 10.

For the PNe listed in the first eleven rows, $T_{\text {OIII }}$ is calculated from the observed [O III] line ratios, while the $T_{\mathrm{NII}}$ is obtained from the temperature relation corresponding to their classes. On the other hand, for the PNe in the last six rows, the opposite occurred, i.e., $T_{\mathrm{NII}}$ comes from the observed [N II] emission-line ratio, which is used in the temperature relation to calculate $T_{\text {OIII }}$ according to the PN class.

Adding the PNe listed in Table 7 to the Martins \& Viegas sample (Table 4), the radial abundance gradients are recalculated. In Table 8, our previous results (rows labeled a1) are compared with the recalculated values (rows labeled a2) for each element. The gradient values $(\alpha)$ are in units of dex/kpc and the abundance at the intercept value $(\beta)$ correspond to the disk abundance extrapolated to the center of the Galaxy. The results are also shown in Figure 5.

For $\mathrm{O}$ and $\mathrm{N}$, the two gradient values show a very good agreement. For $\mathrm{S}$ and $\mathrm{Ne}$, the results are in agreement within the errors. However, recall that, mainly for excitation class 4 , the theoretical relation between the temperatures of the high- and low-ionization zones may perhaps not be a good indicator (see Fig. 3d). Thus, the 

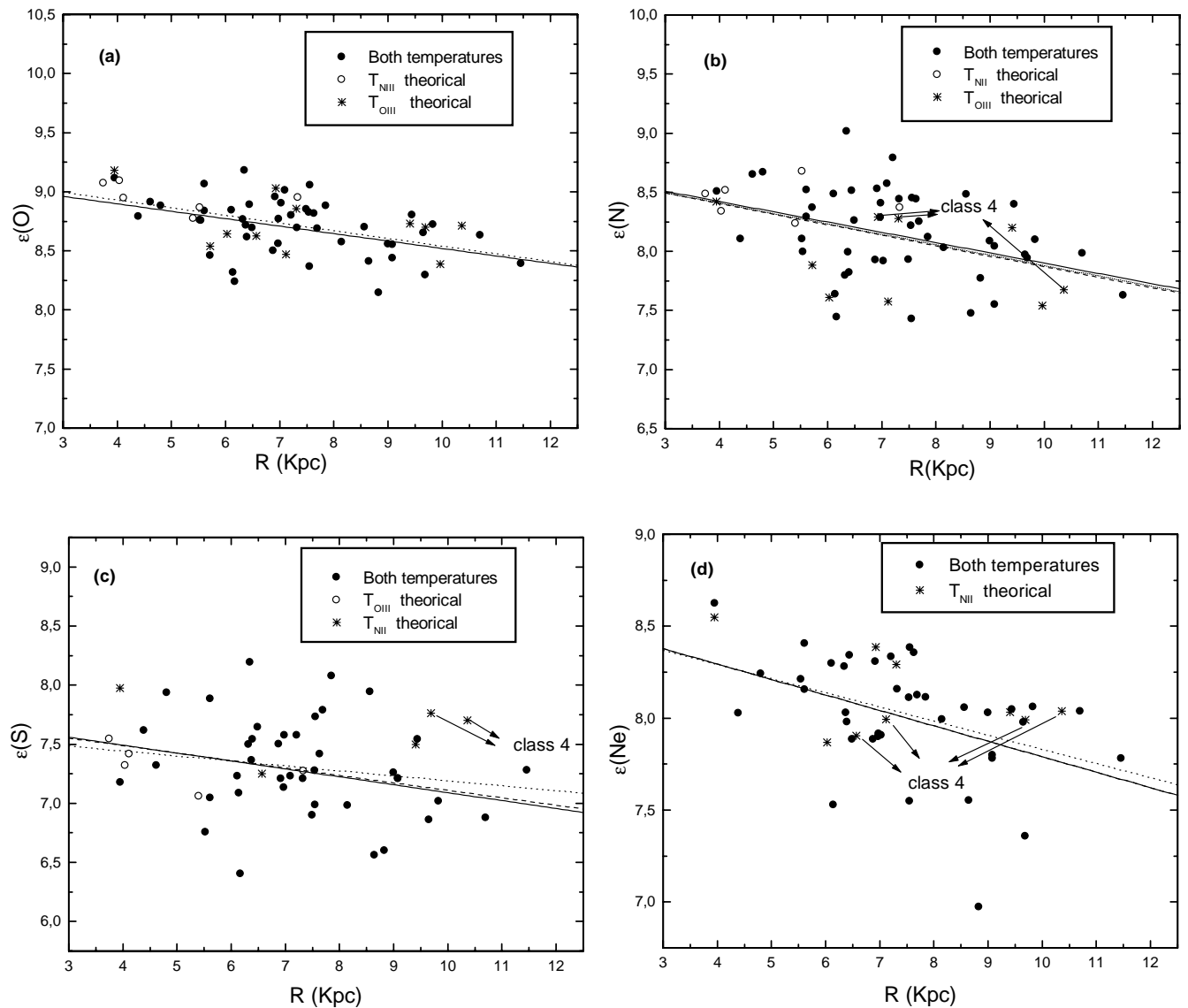

Fig. 5. Abundance gradients of O, N, Ne and S for type II PNe. The solid, dashed and dotted lines correspond, respectively, to (a1) our previous results (Martins \& Viegas 2000), (a2) results including the PNe listed in Table 7, and (a3) results after excluding the class $4 \mathrm{PNe}$ of the complementary sample.

abundances derived for class 4 objects may be wrong, leading to an error in the new gradient value.

There are only 3 class 4 objects in Table 7 , all of them with $T_{\mathrm{NII}}$ obtained from the temperature relation. The N, $\mathrm{Ne}$ and $\mathrm{S}$ abundances are strongly dependent on $T_{\mathrm{NII}}$. To test this issue, a new gradient is calculated, removing class 4 objects from the complementary sample. The results are also shown in Table 8 (rows a3).

For Ne and S, the new results without the class 4 objects show a better agreement with the first values (row 1). On the other hand, class 4 objects seem to have little effect on the nitrogen abundance. This could be due to the fact that only 2 (out of 3 ) class 4 objects were included in the calculation and that coincidentally for both of them the models provide a good fit to the empirical temperatures of the high- and low ionization zones.

\subsection{Types I and III planetary nebulae}

In the case of types I and III PNe, only the oxygen abundance gradient should be used to test the temperature relation. The nitrogen and sulfur abundances are changed by nucleosynthesis of their progenitor stars, and are not characteristic of the interstellar medium (Maciel \& Köppen 1994). In addition, Ne data are available for only few
Table 8. Linear fit of the abundance gradients for type II PNe.

\begin{tabular}{cccccc}
\hline \hline & & $\alpha$ & $\sigma_{\alpha}$ & $\beta$ & $\sigma_{\beta}$ \\
\hline $\mathrm{O}$ & $\mathrm{a} 1$ & -0.063 & 0.018 & 9.15 & 0.14 \\
& $\mathrm{a} 2$ & -0.065 & 0.014 & 9.19 & 0.10 \\
$\mathrm{~N}$ & $\mathrm{a} 1$ & -0.087 & 0.030 & 8.77 & 0.25 \\
& $\mathrm{a} 2$ & -0.088 & 0.024 & 8.76 & 0.17 \\
& $\mathrm{a} 3$ & -0.089 & 0.025 & 8.76 & 0.18 \\
$\mathrm{~S}$ & $\mathrm{a} 1$ & -0.067 & 0.036 & 7.76 & 0.27 \\
& $\mathrm{a} 2$ & -0.042 & 0.028 & 7.61 & 0.21 \\
& $\mathrm{a} 3$ & -0.063 & 0.029 & 7.74 & 0.21 \\
$\mathrm{Ne}$ & $\mathrm{a} 1$ & -0.084 & 0.027 & 8.63 & 0.20 \\
& $\mathrm{a} 2$ & -0.077 & 0.023 & 8.60 & 0.17 \\
& $\mathrm{a} 3$ & -0.084 & 0.024 & 8.63 & 0.18 \\
\hline
\end{tabular}

objects, so the statistical error in the abundance gradient is too large, and the Ne gradient will not provide a good test for the temperature relation.

For types I and III PNe, fewer emission-line spectra are available in the literature. The distances come from Maciel \& Köppen (1994). They were obtained with the same method as in Maciel \& Quireza (1999), but assuming a solar distance of $8.5 \mathrm{kpc}$ from the galactic center, while for type II PNe, $7.6 \mathrm{kpc}$ was adopted. Thus we cannot compare the oxygen gradients derived from type I and III 
Table 10. Linear fit of the abundance gradients for types I and III PNe.

\begin{tabular}{cccccc}
\hline \hline Type I PNe & & $\alpha$ & $\sigma_{\alpha}$ & $\beta$ & $\sigma_{\beta}$ \\
\hline O & $\mathrm{a} 1$ & -0.040 & 0.020 & 9.05 & 0.18 \\
& $\mathrm{a} 2$ & -0.049 & 0.017 & 9.13 & 0.14 \\
\hline Type III PNe & & $\alpha$ & $\sigma_{\alpha}$ & $\beta$ & $\sigma_{\beta}$ \\
\hline $\mathrm{O}$ & $\mathrm{a} 1$ & -0.052 & 0.023 & 8.87 & 0.17 \\
& $\mathrm{a} 2$ & -0.049 & 0.021 & 8.85 & 0.16 \\
\hline
\end{tabular}

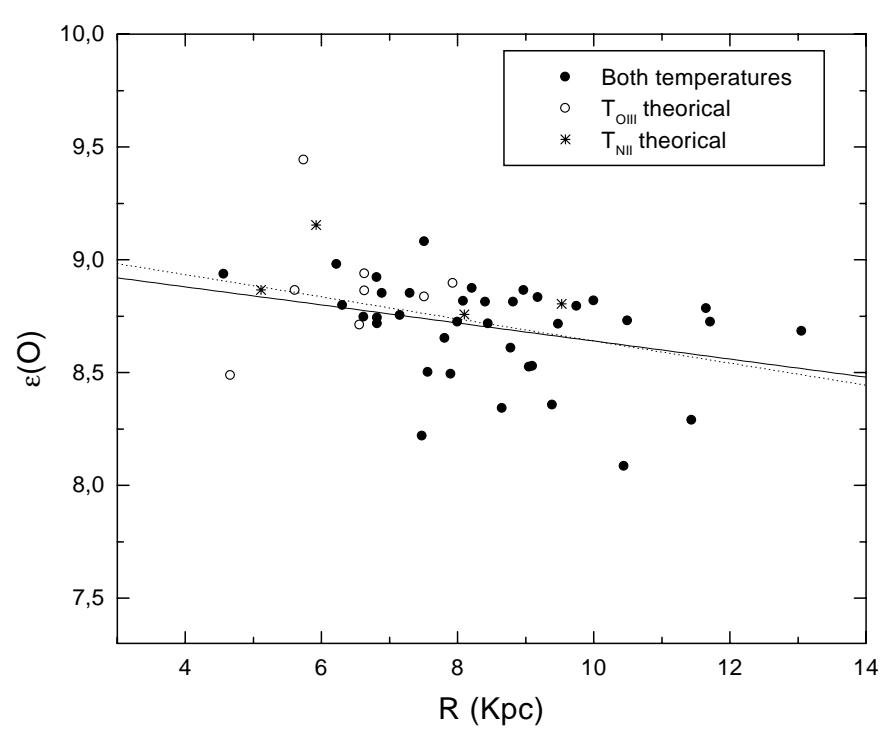

Fig. 6. Oxygen gradient for type I PNe.

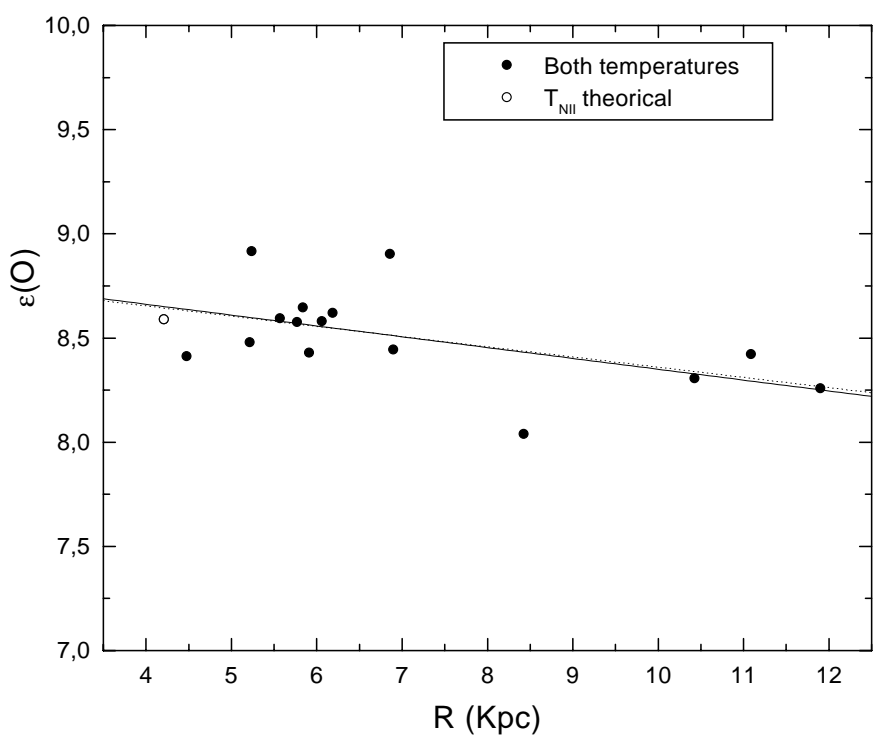

Fig. 7. Oxygen gradient for type III PNe.

PNe with those derived from type II objects. Notice however that, for our purposes, i.e., testing the relation between $T_{\mathrm{NII}}$ and $T_{\mathrm{NII}}$ (Sect. 3 ), this is not a problem.

The same procedure used for type II PNe is adopted for types I and III PNe. The complementary PNe, to be added to the samples presented in Tables 3 and 5, are listed in Table 9
The results for types I and III planetary nebulae presented in Table 10 and in Figs. 6 and 7, respectively, confirm the conclusions reached with type II PNe, i.e., the theoretical temperature relations obtained for different excitation classes of PNe are an efficient tool to overcome the lack of measured auroral line intensities when determining empirical elemental abundances.

\section{Concluding remarks}

Approximations are inevitable when we study a large number of objects, since the individual analysis of each object may be time-consuming and difficult. Empirical methods have been largely used to determine elemental abundances from observed emission-lines. Nevertheless, we have to be careful when assuming the gas physical conditions. For large samples of planetary nebulae it is usual to adopt a temperature of $10^{4} \mathrm{~K}$ and a electron density of $10^{4} \mathrm{~cm}^{-3}$ when the temperature and density indicators obtained from the emission-lines are not available. Depending on the object, these assumptions may induce a large error in the chemical abundances, leading to false conclusions on studies based on those results, like abundance gradients in galaxies or galactic chemical evolution.

For many planetary nebulae, the temperature characterizing the high-ionization zone, $T_{\text {OIII }}$, or the lowionization zone, $T_{\mathrm{NII}}$, cannot be obtained because the temperature indicators are line ratios involving faint auroral lines which are not easily observed. In this paper, we discuss a method to overcome this difficulty for planetary nebulae, establishing a relationship between the two temperatures. Although a correlation is not easily seen when a sample of non-classified PNe is used, the situation is improved when they are gathered into excitation classes.

There are many studies using the PNe excitation classes. The Aller (1956) classification is probably the most used one. More recently, Ratag et al. (1997) suggested that the ratios used in the classification should be calculated with emission-lines of the same element, so that abundances would not influence the results. However, those authors did not present the numerical values that define their excitation classes, so 4 excitation classes are defined and used throughout this paper.

For each of the 4 excitation classes, a linear relation between $T_{\text {OIII }}$ and $T_{\text {NII }}$ is obtained using results from photoionization models which fit the temperatures deduced from the observed emission-line ratios of a sample of types I-III PNe.

The temperature relations, obtained from a grid of photoionization models, are in agreement with the empirical temperatures of a large number of PNe in the sample. On the other hand, most of the objects for which the temperature relation does not provide a good fit to the temperatures deduced from the observations are exotic planetary nebulae, showing uncommon features, or highly ionized class 4 PNe. For all these objects, an individual analysis is necessary. 
In addition, class 1 objects tend to present $T_{\mathrm{NII}}>$ $T_{\text {OIII }}$, in disagreement with the temperature relation deduced from the photoionization models. This fact is probably an indication that shocks or high density condensations are present in these class $1 \mathrm{PNe}$. If the high $T_{\mathrm{NII}}$ is due to shocks, the higher temperature of the postshock zone should favor the low ionization zone where $\mathrm{N}^{+}$is present. On the other hand, if high density condensations are present $\left(\mathrm{n}_{e}\right.$ of the order of $\left.10^{6} \mathrm{~K}\right)$, we expect $T_{\mathrm{NII}}>T_{\mathrm{OIII}}$. In fact, the critical density of the [N II] nebular line is lower than that of the [O III] line, thus due to the density condensations the nitrogen auroral/nebular line ratio would increase more than the oxygen ratio leading to higher empirical temperature in the low-ionization zone.

The method is tested by recalculation of the abundance gradient using a larger number of $\mathrm{PNe}$, i.e., including those objects firstly excluded from the sample because one of the two empirical temperatures was not available. It is worth noting that the large uncertainty in the PNe distances has no effect on the test carried out in this paper. The radial abundance gradient value does not change and the statistical error decreases as expected.

In brief, the results presented here may be improved by more sophisticated models, accounting for non-symmetric geometries, for the presence of condensations and/or for additional heating sources, which have been invoked in the literature to explain the discrepancy between the temperatures derived from the [O III] emission-line ratio and the Balmer discontinuity.

Acknowledgements. We thank Roberto Costa for helpful discussions on the subject. We are also indebted to S. Hyung for the careful reading of this paper and for the suggestions that improved it. This paper was supported by FAPESP(00/12217-4 and 00/06695-0), $\operatorname{CNPq}(303077 / 77-1)$ and PRONEX/Finep(41.96.0908.00).

\section{References}

Aller, L. H. 1956, Gaseous Nebulae, The international Astrophysics Series, vol. III, 66

Aller, L. H., \& Czyzak, S. J. 1983, 51, 211

Aller, L. H., \& Keyes, C. D. 1987, ApJS, 65, 405

Aller, L. H., \& Hyung, S. 1995, MNRAS, 276, 1101

Aller, L. A., Hyung, S., \& Feibelman, W. A. 1995, PNAS, USA, 96, 5366

Aller, L. A., Hyung, S., \& Feibelman, W. A. 1996, PASP, 108, 488

Aller, L. H., Keyes, C. D., \& Feibelman, W. A. 1986, ApJ, 311, 930

Aller, L. H., Keyes, C. D., \& Feibelman, W. A. 1988, PASP, 100, 192

Acker, A., Köppen, J., Stenholm, B., \& Jasniewicz, G. 1989, A\&AS, 80, 201

Balick, B. 1987, AJ, 94, 671

Barker, T. 1978, ApJ, 219, 914

Cannon 1916, Harvard Ann., 76, 20

Feibelman, W. A., Hyung, S., \& Aller, L. H. 1996, MNRAS, 278,625
Feibelman, W. A., Hyung, S., \& Aller, L. H. 1994, ApJ, 426, 653

French, H. B. 1981, ApJ, 246, 434

Freitas-Pacheco, J. A., Maciel, W. J., \& Costa, R. D. D. 1992, A\&A, 261, 579

Gruenwald, R., \& Viegas, S. M. M. 1992, ApJS, 78, 153

Gruenwald, R., \& Viegas, S. M. M. 2000, ApJ, 543, 889

Gurzadyan, G. A., \& Egikyan, A. E. 1991, Ap\&SS, 181, 73

Gutierrez-Moreno, A., Cortes, G., \& Moreno, H. 1985, PASP, 97, 397

Hyung, S. 1994, ApJS, 90, 119

Hyung, S. 1999, JKAS, 32, 55

Hyung, S., \& Aller, L. H. 1993, PASP, 105, 1279

Hyung, S., \& Aller, L. H. 1997a, MNRAS, 292, 71

Hyung, S., \& Aller, L. H. 1997b, ApJ, 491, 242

Hyung, S., \& Aller, L. H. 1998, PASP, 110, 466

Hyung, S., Aller, L. H., \& Feibelman, W. A. 1994a, MNRAS, 269,975

Hyung, S., Aller, L. H., \& Feibelman, W. A. 1994b, PASP, 106, 745

Hyung, S., Aller, L. H., \& Feibelman, W. A. 1997, ApJS, 108, 5030

Hyung, S., Aller, L. H., \& Feibelman, W. A. 1999a, ApJ, 514, 878

Hyung, S., Aller, L. H., \& Feibelman, W. A. 1999b, ApJ, 525, 294

Hyung, S., Aller, L. H., Feibelman, W. A., \& Lee, W.-B. 2001, AJ, 122, 954

Hyung, S., Aller, L. H., \& Lee, W.-B. 2001, PASP, 113, 1559

Hyung, S., Keyes, C. D., \& Aller, L. H. 1994c, MNRAS, 272, 49

Huggins, W. 1864, MNRAS, 25, 60

Kaler, J. B., Kwitter, K. B., Shaw, R. A., \& Browning, L. 1996 , PASP, 108, 980

Kaler, J. B., Shaw, R. A., \& Browning, L. 1997, PASP, 109, 289

Kaler, J. B., Shaw, R. A., Feibelman, W. A., \& Imhoff, C. L. 1991, PASP, 103, 67

Kaler, J. B., Shaw, R. A., \& Kwitter, K. B. 1990, ApJ, 359, 392

Kaller, J. B., Stanghellini, L., \& Shaw, A. 1993, A\&A, 279, 529

Kingsburgh, R. L., \& Barlow, M. J. 1994, MNRAS, 271, 253

Maciel, W. J. 1984, A\&AS, 55, 253

Maciel, W. J., \& Köppen, J. 1994, A\&A, 282, 436

Maciel, W. J., \& Quireza, C. 1999, A\&A, 345, 629

Martins, L. P., \& Viegas, S. M. M. 2000, A\&A, 361, 1121

Meaburn, J., Clayton, C. A., Bryce, M., \& Walsh, J. R. 1996, MNRAS, 281, 57

Moreno, H., Gutierrez-Moreno, A., Cortes, G., \& Hamuy, M. 1994, PASP, 106, 619

Page 1942, ApJ, 96, 78

Pagel, B. E. J. 1992, IAU Symp. 149, ed. B. Barbuy, \& A. Renzini, 133

Peimbert, M., \& Costero, R. 1969, Bol. Obs. Tonantzintla y Tacubaya, 5, 3

Peimbert, M., \& Torres-Peimbert, S. 1987, Rev. Mex. Astron. Astrofís., 14, 540

Phillips, J. P., \& Cuesta 1998, A\&AS, 133, 381

Phillips, J. P., \& Cuesta, L. 1999, AJ, 118, 2929

Ratag, M. A., Pottasch, S. R., Dennefeld, M., \& Menzies, J. 1997, A\&AS, 126, 297

Sabbadin, F. 1984, MNRAS, 210, 341

Stasinska, G. 1990, A\&AS, 83, 501

Tamura, S., \& Shaw, R. A. 1987, PASP, 99, 1264

Webster, B. L 1988, MNRAS, 230, 377 